Moore Luke (Orcid ID: 0000-0003-4481-9862)

Cravens Thomas, E. (Orcid ID: 0000-0003-0912-8353)

Mueller-Wodarg Ingo, C. F. (Orcid ID: 0000-0001-6308-7826)

Perry Mark, E (Orcid ID: 0000-0003-1600-6856)

Waite Jack, Hunter (Orcid ID: 0000-0002-1978-1025)

Perryman Rebecca (Orcid ID: 0000-0003-1858-4692)

Mitchell Donald, G. (Orcid ID: 0000-0003-1960-2119)

Persoon Ann, M. (Orcid ID: 0000-0001-8161-2225)

Wahlund Jan-Erik (Orcid ID: 0000-0002-2107-5859)

Morooka Michiko, W. (Orcid ID: 0000-0001-9958-0241)

\title{
Models of Saturn's equatorial ionosphere based on in situ data from Cassini's Grand Finale
}

\author{
L. Moore ${ }^{1}$, T. E. Cravens ${ }^{2}$, I. Müller-Wodarg ${ }^{3}$, M. E. Perry ${ }^{4}$, J. H. Waite ${ }^{5}$, R. Perryman ${ }^{5}$, A.

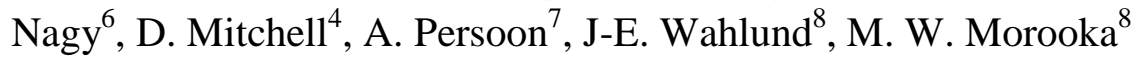 \\ ${ }^{I}$ Center for Space Physics, Boston University, Boston, Massachusetts, USA \\ ${ }^{2}$ Department of Physics and Astronomy, University of Kansas, Lawrence, Kansas, USA \\ ${ }^{3}$ Blackett Laboratory, Imperial College London, Prince Consort Road, London SW7 2AZ, UK \\ ${ }^{4}$ Johns Hopkins University Applied Physics Laboratory, Laurel, Maryland, USA \\ ${ }^{5}$ Southwest Research Institute, San Antonio, Texas, USA \\ Department of Climate and Space Sciences and Engineering, University of Michigan, Ann Arbor, Michigan, USA \\ ${ }^{7}$ Department of Physics and Astronomy, University of Iowa, Iowa City, Iowa, USA \\ ${ }^{8}$ Swedish Institute of Space Physics, Box 537, SE-751 21 Uppsala, Sweden
}

Corresponding author: Luke Moore (moore@bu.edu)

\section{Key points:}

- Unexpectedly complex influx of ring material found in Saturn's equatorial upper atmosphere, including organics, water and nanograins.

- Ring influx leads to reduction in major ions $\left(\mathrm{H}^{+}\right.$and $\left.\mathrm{H}_{3}{ }^{+}\right)$; heavier molecular ions dominate Saturn's low-altitude equatorial ionosphere.

- Major molecular ions at low-altitude still uncertain, but are likely to include $\mathrm{H}_{3} \mathrm{O}^{+}$and $\mathrm{HCO}^{+}$, and the mean modeled ion mass is $11 \mathrm{Da}$.

This is the author manuscript accepted for publication and has undergone full peer review but has not been through the copyediting, typesetting, pagination and proofreading process, which may lead to differences between this version and the Version of Record. Please cite this article as doi: 10.1029/2018GL078162

This article is protected by copyright. All rights reserved. 
Latest edit: 28 June 2018

Submitted: $\quad 30$ March 2018

Revised: $\quad 21$ May 2018

Accepted:

\section{Abstract}

We present new models of Saturn's equatorial ionosphere based on the first in situ measurements of its upper atmosphere. The neutral spectrum measured by Cassini's Ion and Neutral Mass Spectrometer (INMS), which includes substantial methane, ammonia, and organics in addition to the anticipated molecular hydrogen, helium, and water, serves as input for unexpectedly complex ionospheric chemistry. Heavy molecular ions are found to dominate Saturn's equatorial lowaltitude ionosphere, with a mean ion mass of 11 Da. Key molecular ions include $\mathrm{H}_{3} \mathrm{O}^{+}$and $\mathrm{HCO}^{+}$; other abundant heavy ions depend upon the makeup of the mass 28 neutral species, which cannot be uniquely determined. INMS neutral species lead to generally good agreement between modeled and observed plasma densities, though poor reproduction of measured $\mathrm{H}^{+}$and $\mathrm{H}_{3}{ }^{+}$ variability and an overabundance of modeled $\mathrm{H}_{3}{ }^{+}$potentially hint at missing physical processes in the model, including a loss process that affects $\mathrm{H}_{3}{ }^{+}$but not $\mathrm{H}^{+}$.

\section{Plain Language Summary}


Cassini's Grand Finale enabled the first-ever direct measurements of Saturn's upper atmosphere. Here, we use Cassini's unique measurements to construct new models of the plasma in this important boundary region that separates the dense lower atmosphere from space. Based on the complex array of observed gases, we find that heavy molecular ions are dominant near Saturn's equator. This surprising result demonstrates that the chemistry in Saturn's equatorial upper atmosphere is substantially more complex than anticipated. The presence of these unexpected ions potentially represents a new method of monitoring Saturn's ionosphere remotely. Furthermore, as other Cassini measurements indicate that the complex chemistry is likely driven by an influx of ring-derived material, such observations may even help to track the evolution of Saturn's rings as they lose mass to its atmosphere. 


\section{Introduction}

Early theoretical models of Saturn's ionosphere predicted peak electron densities, $\mathrm{N}_{\mathrm{MAX}}$, of $10^{5} \mathrm{~cm}^{-3}$ on the dayside (e.g., McElroy, 1973; Waite et al., 1979), significantly larger than the $\sim 10^{4} \mathrm{~cm}^{-3}$ found from subsequent radio occultation measurements by the Pioneer 11 (Kliore et al., 1980) and Voyager spacecraft (Lindal et al., 1985). The Cassini spacecraft obtained an additional 59 radio occultations of Saturn's ionosphere, re-confirming the average $\mathrm{N}_{\mathrm{MAX}}$ value of $\sim 10^{4} \mathrm{~cm}^{-3}$, as well as finding a dawn/dusk asymmetry (Nagy et al., 2006) and an unexpected latitudinal trend in $\mathrm{N}_{\mathrm{MAX}}$ (Kliore et al., 2009, 2014). Therefore, in order to reproduce observations by reducing modeled electron densities, modelers have long considered different methods for converting $\mathrm{H}^{+}$- a dominant and long-lived atomic ion - into a short-lived molecular ion. The most commonly considered pathways for chemical loss of $\mathrm{H}^{+}$involved charge exchange with (1) vibrationally excited $\mathrm{H}_{2}$ (e.g., McElroy, 1973; Atreya et al., 1984), and (2) water products, likely introduced from Saturn's rings and/or icy moons (Shimizu, 1980; Chen, 1983; Connerney and Waite, 1984). Uncertainties in the $\mathrm{H}^{+}+\mathrm{H}_{2}$ reaction rate and the $\mathrm{H}_{2}$ vibrational population have rendered the former process dependent upon estimates of poorly known sources and sinks of $\mathrm{H}_{2}$ vibrational levels (e.g., Cravens, 1987; Majeed et al., 1991). Constraints on the latter process, on the other hand, have evolved with an increasingly comprehensive set of observations and model comparisons (summarized in Moore et al., 2017), and have crystallized in Cassini's measurements of an influx of ring material near Saturn's equator (Hsu et al., 2018; Mitchell et al., 2018; Perry et al., 2018; Waite et al., 2018). 
The first in situ measurements of Saturn's upper atmosphere were obtained by the Cassini spacecraft during the proximal orbit segment (spanning April - September 2017) of the Grand Finale phase of its 13-year mission. These data find evidence for a strong influence of Saturn's rings on its equatorial upper atmosphere, manifesting as an influx of grains and related material. Observations of grains within Saturn's upper atmosphere and exosphere are described by Mitchell et al. (2018) and Hsu et al. (2018). An overview of two different methods by which the Radio and Plasma Wave Science (RPWS) instrument determines electron density $\left(\mathrm{n}_{\mathrm{e}}\right)$ along Cassini's trajectory is given in Morooka et al. (2018) and Persoon et al. (2018). Measurements of neutral and ion densities in Saturn's upper atmosphere by the Ion and Neutral Mass Spectrometer (INMS), as well as initial ionospheric interpretations, are summarized by Waite et al. (2018) and Cravens et al. (2018). Here, using the constraints provided by all of the preceding data, we present new models of Saturn's equatorial ionosphere.

\section{An influx of external material: past evidence and ionospheric effect}

Interplanetary dust grains represent an important source of exogenous material for all of the giant planets. Recently updated interplanetary dust fluxes in the outer Solar System (Poppe, 2016), combined with a dust ablation model, are able to explain a wide range of observational constraints of stratospheric composition, though with a few notable exceptions (Moses and Poppe, 2017). The most obvious exception is at Saturn, where the interplanetary dust grain flux is more than an order of magnitude too small to explain the observed stratospheric $\mathrm{H}_{2} \mathrm{O}$, $\mathrm{CO}$ and 
$\mathrm{CO}_{2}-\mathrm{a}$ discrepancy easily explained, at least qualitatively, by Saturn's vast ring system and its active cryovolcanic moon, Enceladus. Nevertheless, despite ample evidence of external material being present in the Saturn system, constraining the source and spatial/temporal variability of any influxes into Saturn's upper atmosphere has proven difficult.

Analysis of early International Ultraviolet Explorer observations led to the suggestion of a possible external source of water or oxygen (Winkelstein et al., 1983). This suggestion was later confirmed with the detection of stratospheric water by the Infrared Space Observatory (Feuchtgruber et al., 1997). Circumstantial evidence also began to mount for a spatial variability to the implied oxygen influxes. First, the latitudinal trends in $n_{e}$ obtained from Cassini radio occultation observations (Kliore et al., 2009, 2014) were best reproduced using an influx peaked at Saturn's equator (Moore et al., 2010). Second, there were indications of localized water enhancements at mid-latitudes. In the UV, a $2 \sigma$-detection of water near $33^{\circ} \mathrm{S}$ was made using the Hubble Space Telescope (Prangé et al., 2006). In the IR, ground-based observations of non-solar latitudinal variations in $\mathrm{H}_{3}{ }^{+}$emission, mirrored in conjugate hemispheres, were found to map along magnetic field lines to regions of expected water influx in Saturn's ring plane (O'Donoghue et al., 2013, 2017), likely evidence for a ring-atmosphere interaction wherein charged ring particles "rain" down along magnetic field lines and alter local ionospheric chemistry (Connerney, 2013; Moore et al., 2015).

Models of Saturn's ionosphere have commonly employed a water influx to modify calculated electron densities via interactions with the major ions, $\mathrm{H}^{+}$and $\mathrm{H}_{3}{ }^{+}$(e.g., Connerney 
and Waite, 1984; Majeed and McConnell, 1991; Moses and Bass, 2000; Kim et al., 2014). In the presence of water, $\mathrm{H}^{+}$ions can be readily converted into short-lived molecular ions, e.g.:

$$
\begin{array}{lll}
\mathrm{H}^{+}+\mathrm{H}_{2} \mathrm{O} & \rightarrow & \mathrm{H}_{2} \mathrm{O}^{+}+\mathrm{H} \\
\mathrm{H}_{2} \mathrm{O}^{+}+\mathrm{H}_{2} & \rightarrow & \mathrm{H}_{3} \mathrm{O}^{+}+\mathrm{H}
\end{array}
$$

The dissociative recombination rate of $\mathrm{H}_{3} \mathrm{O}^{+}$with electrons is $>10^{5}$ times faster than the radiative recombination of $\mathrm{H}^{+}$, and therefore the modeled $n_{e}$ in such an ionosphere is smaller, provided the influx of water is sufficient. There is, in addition, a secondary effect: as dissociative recombination with electrons is the primary loss for $\mathrm{H}_{3}{ }^{+}$, an introduction of water will reduce the $\mathrm{H}_{3}{ }^{+}$loss rate, thereby increasing $\mathrm{H}_{3}{ }^{+}$density (Moore et al., 2015). However, for extremely large influxes $\left(>2 \times 10^{7} \mathrm{H}_{2} \mathrm{O}\right.$ molecules $\left.\mathrm{cm}^{-2} \mathrm{~s}^{-1}\right)$ this secondary effect is overwhelmed by loss of $\mathrm{H}_{3}^{+}$ via a charge-exchange reaction similar to (1).

The facts that Saturn's rings are mostly water ice (Cuzzi et al., 2010) and that the Enceladus plumes are mostly water vapor (Waite et al., 2006) made it natural to assume that the dominant influx into Saturn's atmosphere was water in some form. Nevertheless, the reduction in $n_{e}$ required for ionospheric models to reproduced observations can be achieved just as easily via $\mathrm{H}^{+}$charge-exchange with methane and the other molecules detected by INMS (Waite et al., 2018).

\section{Modeling approach}




\subsection{New constraints based on Cassini in situ data}

Cassini in situ measurements offer the first opportunity to simultaneously constrain neutral and plasma parameters in Saturn's upper atmosphere, a significant advance for ionospheric models. In addition, INMS measurements of $\mathrm{H}_{2}^{+}$can be used to empirically determine the photoionization rate - a parameter that can be difficult to ascertain due to sparse solar EUV data at Saturn. Under photochemical equilibrium (PCE), ion production and loss rates for each species $s$ are locally equal (i.e., $P_{s}=L_{s}$ ), and ion densities can be calculated analytically (Schunk and Nagy, 2009). For PCE to hold, the chemical lifetime of a species must be much shorter than the transport timescale. This assumption holds for altitudes below $\sim 2500 \mathrm{~km}$ for conditions of Cassini's closest approach during the proximal orbits (slightly higher than for Saturn's mid-latitudes, as calculated by Moore et al., 2004). For $\mathrm{H}_{2}{ }^{+}$, production is given by $P_{H_{2}^{+}}=j n_{H_{2}}$, where $j$ is the ionization frequency $\left(\mathrm{s}^{-1}\right)$, which depends on the incident solar EUV flux and the photoionization cross-section of $\mathrm{H}_{2}$, and $n_{\mathrm{H}_{2}}$ is the $\mathrm{H}_{2}$ number density $\left(\mathrm{cm}^{-3}\right)$. The dominant loss for $\mathrm{H}_{2}{ }^{+}$ions, and source of $\mathrm{H}_{3}{ }^{+}$ions, is the rapid charge-exchange reaction

$$
\mathrm{H}_{2}^{+}+\mathrm{H}_{2} \quad \rightarrow \quad \mathrm{H}_{3}^{+}+\mathrm{H} \quad k_{3}=2 \times 10^{-9} \mathrm{~cm}^{3} \mathrm{~s}^{-1}
$$

The loss of $\mathrm{H}_{2}{ }^{+}$is therefore given by $L_{H_{2}^{+}}=k_{3} n_{H_{2}} n_{H_{2}^{+}}$; equating production and loss gives:

$$
j=k_{3} n_{H_{2}^{+}}
$$


Reaction rate $k_{3}$ is well-known (Anicich, 1993), and so the INMS-measured $\mathrm{H}_{2}{ }^{+}$densities at the top of the atmosphere (i.e., where optical depth is minimal) give a firm constraint on $j$. For orbit $\mathrm{P} 288$, on 14 August 2017, where the peak $\mathrm{H}_{2}^{+}$density is $\sim 0.6 \mathrm{~cm}^{-3}, j$ is $\sim 1.2 \times 10^{-9} \mathrm{~s}^{-1}$.

INMS was able to measure ion densities on four proximal orbits: two with a closest approach near $3000 \mathrm{~km}$ above the 1 bar pressure level (P283 and P287 on 12 July 2017 and 7 August 2017, respectively), and two with a closest approach near $1700 \mathrm{~km}$ altitude (P288 and P292 on 14 August 2017 and 9 September 2017, respectively), between Saturn's homopause and exobase. Figure 1 shows Cassini results for P288 and P292, from which we can derive a number of constraints to guide the model comparisons. First, the INMS ion densities detected $\left(\mathrm{H}^{+}, \mathrm{H}_{2}^{+}, \mathrm{H}_{3}^{+}\right.$, and $\left.\mathrm{He}^{+}\right)$are represented by gray, dark red, blue, and gold curves, and the sum of those light ions is given by the black dotted curve. Ion densities in Figure 1a are adapted from those presented in Waite et al. (2018), and follow from the sensitivities discussed in Cravens et al. (2018). Figure $1 \mathrm{~b}$ presents the P292 densities using the same analysis. Second, electron densities obtained from RPWS Langmuir Probe (LP) sweep data and from plasma wave analyses are shown by the orange and turquoise curves, respectively. Uncertainties for these measurements are <20\% (Morooka et al., 2018) and 9-19\% (Persoon et al., 2018). Third, the response of the Magnetospheric IMaging Instrument's Charge Energy Mass Spectrometer (MIMI/CHEMS) to dust grain impact is scaled by a constant factor, added to the total INMS ion density, and plotted in pink (Mitchell et al., 2018). Finally, in order to give context to the plasma densities, panels (c) and (d) present a number of relevant INMS neutral densities over the same 
region: $\mathrm{H}_{2}, \mathrm{HD}, \mathrm{He}$, and $\mathrm{CH}_{4}$ are represented by dark red, cyan, gold, and tan curves, respectively. These densities follow from the approach discussed in Perry et al. (2018).

Panels 1c and 1d provide in situ neutral density constraints for the ionospheric modeling described below. In addition, they are used to guide extrapolation to altitudes above and below the Cassini trajectory, such that a self-consistent neutral background atmosphere can be used as a base for ionospheric calculations. This extrapolation proceeds in a series of steps. First, representative temperatures are derived by fitting an isothermal model to the measured $\mathrm{H}_{2}$ densities. This approach is described by Yelle et al. (2018), and we adopt $370 \mathrm{~K}$ based on their values: $368.8 \pm 1.1 \mathrm{~K}$ and $372.1 \pm 1.0 \mathrm{~K}$ for P288 and P292, respectively. Second, densities for each neutral constituent are extrapolated in altitude based on scale heights appropriate for these exospheric temperatures. The mixing ratios of methane and other heavy molecular species are found to be roughly constant in the region of the thermosphere sampled by Cassini (i.e., above the homopause; Waite et al., 2018), consistent with a topside influx of a minor species. The $\mathrm{H}_{2}$ scale height is therefore used for their density extrapolations. Finally, $\mathrm{H}$ is also included by using a modeled mixing ratio at Cassini's altitude (Müller-Wodarg et al., 2012), and then extrapolating it in altitude according to its mass-appropriate scale height. 

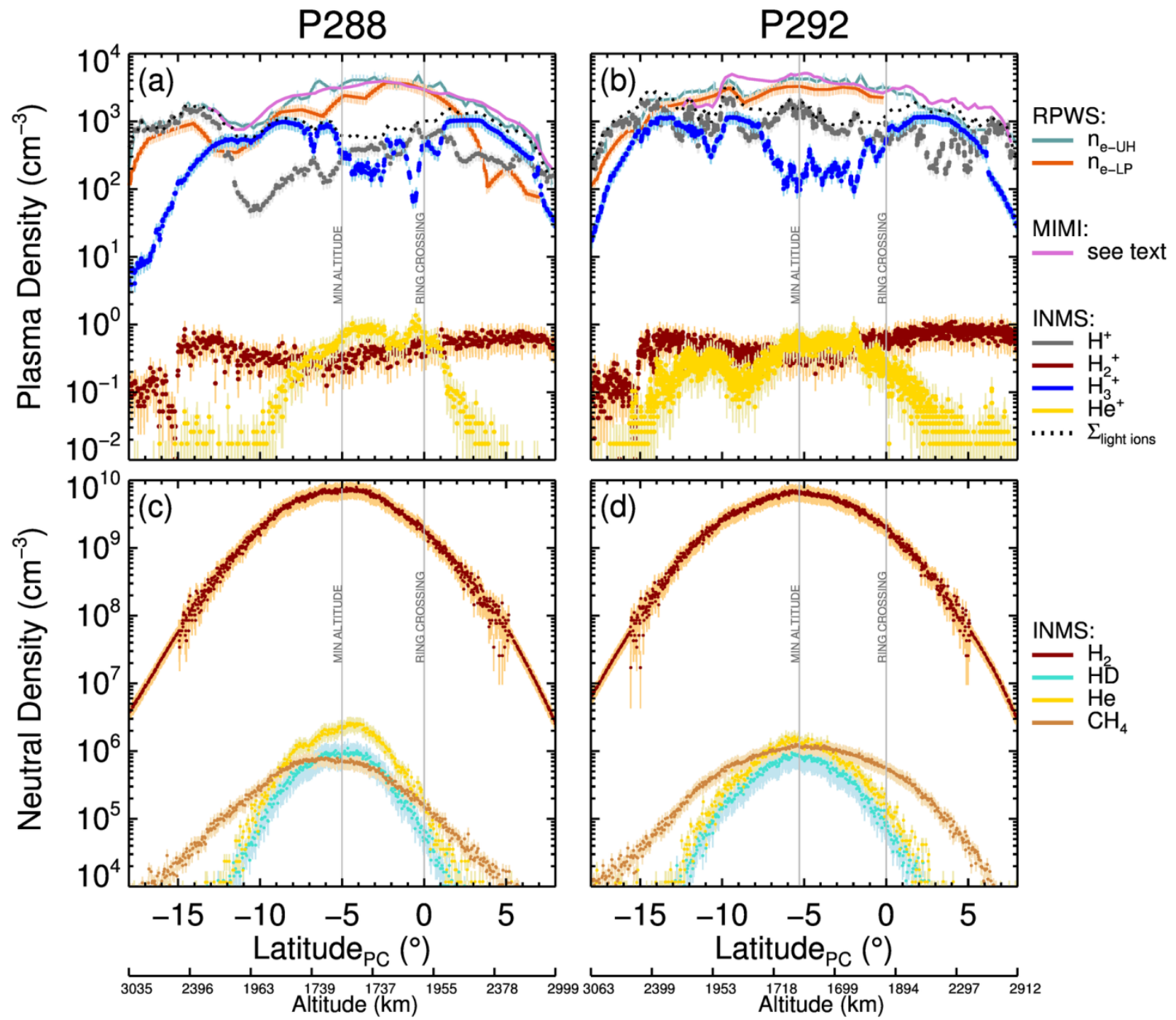

Figure 1: Data comparisons from the INMS, RPWS and MIMI/CHEMS instruments for proximal orbits (a and c) 288, and (b and d) 292. Cassini sampled the upper atmosphere near local solar noon at closest approach. The minimum altitude and the ring plane crossing for each orbit are indicated by gray vertical lines. Panel (a) is adapted from Waite et al. (2018).

There are a number of points to highlight from the data presented in Figure 1. In terms of magnitudes, the P288 and P292 structures are fairly similar. However, there is clearly significant variability also present in Saturn's equatorial ionosphere. This variability is perhaps 
most obvious in the ion densities, though there are also differences in the neutral structure for these two passes that sample a nearly identical range of altitudes, latitudes and local times. Solar EUV irradiance would not give rise to the narrow fluctuations especially evident in the $\mathrm{H}^{+}, \mathrm{H}_{3}{ }^{+}$ and $n_{e}$ densities. Therefore, the observed variability might be a signature of the effect of dust and other ring material on Saturn's ionosphere (e.g., Waite et al., 2018), or possibly a reflection of the narrow layers in electron density frequently observed by radio occultations (Kliore et al., 2009). The effects of the attenuation of sunlight by Saturn's rings - "ring shadowing" - start to become a factor at latitudes below $\sim 7^{\circ}$ south (Moore et al., 2004), and the precipitous drop in $\mathrm{H}_{2}{ }^{+}$ near $-15^{\circ}$ corresponds to the shadow of the optically-thick inner B ring. Similar signatures of ring shadowing are present in $\mathrm{H}^{+}, \mathrm{H}_{3}{ }^{+}$, and $n_{e}$ densities, though they are much less obvious due to the more complicated evolution of those species (Wahlund et al., 2017; Hadid et al., 2018; Waite et al., 2018).

There is a significant discrepancy between the measured electron density and the sum of the light ion densities, peaking near closest approach, with the former larger than the latter by factors up to $\sim 8(\mathrm{P} 288)$ and $\sim 5(\mathrm{P} 292)$, respectively. If charge neutrality is assumed, then this discrepancy implies that the bulk of the ions in Saturn's low-altitude $(<\sim 2100 \mathrm{~km})$ equatorial ionosphere are heavier than 7 Daltons, as the high speed of the Cassini spacecraft during the proximal orbits meant that INMS was only able sample ions with mass numbers $<8 \mathrm{Da}$ (and over that range only $\mathrm{H}^{+}, \mathrm{H}_{2}{ }^{+}, \mathrm{H}_{3}{ }^{+}$, and $\mathrm{He}^{+}$were detected, as expected; Cravens et al., 2018). RPWS/LP measurements support this implication, finding a predominance of heavy ions near 
closest approach (Wahlund et al., 2017). The complex assortment of neutrals detected by INMS - including methane $\left(\mathrm{CH}_{4}\right)$, water $\left(\mathrm{H}_{2} \mathrm{O}\right)$, carbon dioxide $\left(\mathrm{CO}_{2}\right)$, ammonia $\left(\mathrm{NH}_{3}\right)$, and an unknown combination of $28 \mathrm{Da}$ species (Waite et al., 2018) - mean that there is abundant material to charge-exchange with the ambient $\mathrm{H}^{+}$and $\mathrm{H}_{3}{ }^{+}$ions, leading to a mix of heavy ions. Such heavy ions could easily explain the gap between the total measured ion and electron densities near closest approach. Furthermore, the good agreement between the electron density structure and the scaled MIMI/CHEMS structure along the Cassini trajectory is evidence for these processes being related, perhaps with the grains acting as a tracer of a more abundant influx or with the grain charge balance affecting the availability of free electrons (Morooka et al., 2018). Taken together, the Cassini INMS, RPWS, and MIMI data indicate that Saturn's equatorial ionosphere is being transformed by an influx of apparently ring-derived material. While previous models predicted the main ionospheric peak would be dominated by $\mathrm{H}^{+}$and $\mathrm{H}_{3}{ }^{+}$, it is clear from the Cassini proximal data that, at least in the equatorial region, Saturn's ionosphere is instead dominated by some combination of heavy ions (Cravens et al., 2018).

\subsection{The Saturn Thermosphere Ionosphere Model (STIM): Cassini-derived inputs}

The Saturn Thermosphere Ionosphere Model (STIM) is a suite of 1-D, 2-D, and 3-D models of Saturn's upper atmosphere. STIM's core is a 3-D global circulation model (GCM) that treats the global response of Saturn's thermosphere and ionosphere to solar and magnetic forcing (Müller-Wodarg et al., 2012). For more localized and/or unconstrained applications 
where use of the GCM is computationally prohibitive, a 1-D ionospheric module that solves the ion continuity and momentum equations over a fixed neutral background is instead used (e.g., Moore et al., 2015 and references therein). We use such an approach here, as it is well-suited for the current work, which consists of making model comparisons with Cassini ionospheric measurements where the neutral atmosphere is fixed by INMS data, and where the ionosphere near closest approach is in PCE (Cravens et al., 2018).

Ion production rates in STIM follow from the attenuation of solar photons (Galand et al., 2009), which are specified here using the EUV and soft X-ray spectrum from the Thermosphere Ionosphere Mesosphere Energetics and Dynamics Solar EUV Experiment (TIMED/SEE: Woods et al., 2005). SEE solar spectra appropriate for P288 and P292 are selected and extrapolated to Saturn's orbital distance based on the angular separation between the Earth and Saturn and the solar rotation rate. This approach is imperfect, and inherently assumes that the solar activity has not changed significantly over the period of solar rotation, but it is the best solution when no in situ solar irradiance data are available. In the present work, however, $\mathrm{H}_{2}{ }^{+}$densities from INMS can be used to constrain the solar ion production rate. In order to reproduce the observed $\mathrm{H}_{2}{ }^{+}$ densities at high altitude, the solar flux at Saturn derived from TIMED/SEE measurements had to be enhanced by $50 \%$ for P288 and 70\% for P292. This correction is likely representative of some combination of (a) the solar flux extrapolation, (b) the incomplete calibration of the post2012 degradation of the SEE instrument (Girazian and Withers, 2015; Huang et al., 2017), and possibly (c) missing ionization due to photoelectrons and associated secondary electrons. 
Process (c) was excluded from the current calculations, as it is not expected to be important above $\sim 1500 \mathrm{~km}$ (Galand et al., 2009; Moore et al., 2009), though future fully-coupled calculations should test this conclusion given the influx of ring material. Regardless of the ultimate source of the ionization correction, the fact that it was needed emphasizes the considerable value of the in situ data in generating model comparisons.

\section{Results and Discussion}

\subsection{Domination by heavy molecular ions}

Figure 1 demonstrates that light ions are a minority in Saturn's low-altitude equatorial ionosphere. In order to explain the relatively minor contribution of $\mathrm{H}^{+}$and $\mathrm{H}_{3}{ }^{+}$ions in this regime, a high abundance $\left(\sim 10^{-4}\right.$ mixing ratio) of heavy molecular species must also be present (Cravens et al., 2018). INMS has measured a number of abundant neutral species that are consistent with this requirement, but there is uncertainty in their identification, particularly for the $28 \mathrm{Da}$ species. Therefore, there is also uncertainty in the corresponding ion composition.

In order to provide bounds on the likely heavy molecular ions present, Figure 2 shows model ionospheres at $6^{\circ} \mathrm{S}$ that follow from the measured neutral densities. For these calculations, we make the assumption that the 28 Da species is entirely (a) $\mathrm{N}_{2}$, (b) $\mathrm{CO}$, or (c) $\mathrm{C}_{2} \mathrm{H}_{4}$. As the mass 28 (non- $\mathrm{H}_{2}$, non-He) mixing ratio varies between $<5 \%$ and $15 \%$ in Table 1 of Waite et al. (2018), we set its density to $40 \%$ of the maximum measured $\mathrm{CH}_{4}$ value (i.e., $\sim 0.15 / 0.35$, where 0.35 is the suggested maximum fraction of $\mathrm{CH}_{4}$ among the non- $\mathrm{H}_{2}$, non-He constituents). 
Densities for $\mathrm{NH}_{3}$ and $\mathrm{CO}_{2}$ are specified in a similar way, using $50 \%$ and $25 \%$ of the $\mathrm{CH}_{4}$ value, respectively. Meanwhile, $\mathrm{H}_{2} \mathrm{O}$ is fixed near its minimum estimated value at $25 \%$ of the measured $\mathrm{CH}_{4}$; this helps more clearly isolate the impact of the unknown $28 \mathrm{Da}$ species. Figure $2 \mathrm{~d}$ presents the corresponding neutral density profiles used in the calculations, with the approximate altitude of the Cassini INMS measurement - indicated by a gray line - providing an anchor for extrapolation in altitude, as described above. As much of Saturn's low-altitude ionosphere is in PCE, the in situ neutral densities could in principle be used to calculate ion densities, avoiding any extrapolation in altitude. However, inclusion of a representative background atmosphere allows for a more accurate calculation of the attenuation of solar photons with altitude, and therefore improved calculation of ion production at Saturn's lowaltitudes. This drop in ion production at low-altitudes is evident in the $\mathrm{H}_{2}{ }^{+}$profiles in Figure 2.

Figure 2 illustrates that, due to the influx of ring material, Saturn's ionospheric chemistry is much more complicated than expected above the homopause (e.g., Kim et al., 2014). Collectively, heavy molecular ions dominate below $\sim 1800 \mathrm{~km}$ altitude, with $\mathrm{H}_{3} \mathrm{O}^{+}$playing a major role in each case. If $\mathrm{CO}$ is a major component of the $28 \mathrm{Da}$ species, then $\mathrm{HCO}^{+}$also becomes a major ion. For the case where the $28 \mathrm{Da}$ species is $\mathrm{N}_{2}, \mathrm{~N}_{2} \mathrm{H}+$ also begins to play a major role, joined by $\mathrm{HCO}_{2}{ }^{+}, \mathrm{CH}_{3}{ }^{+}$and $\mathrm{CH}_{5}{ }^{+}$. Finally, if the 28 Da neutral is instead entirely $\mathrm{C}_{2} \mathrm{H}_{4}$, then the chemistry is noticeably more complex and the lower ionosphere populated by a number of hydrocarbon ions (e.g., $\mathrm{C}_{2} \mathrm{H}_{3}{ }^{+}, \mathrm{CH}_{3}{ }^{+}, \mathrm{C}_{2} \mathrm{H}_{5}{ }^{+}$, and $\mathrm{C}_{3} \mathrm{H}_{5}{ }^{+}$) as well as $\mathrm{HCO}^{+}$and $\mathrm{HCO}_{2}{ }^{+}$. 

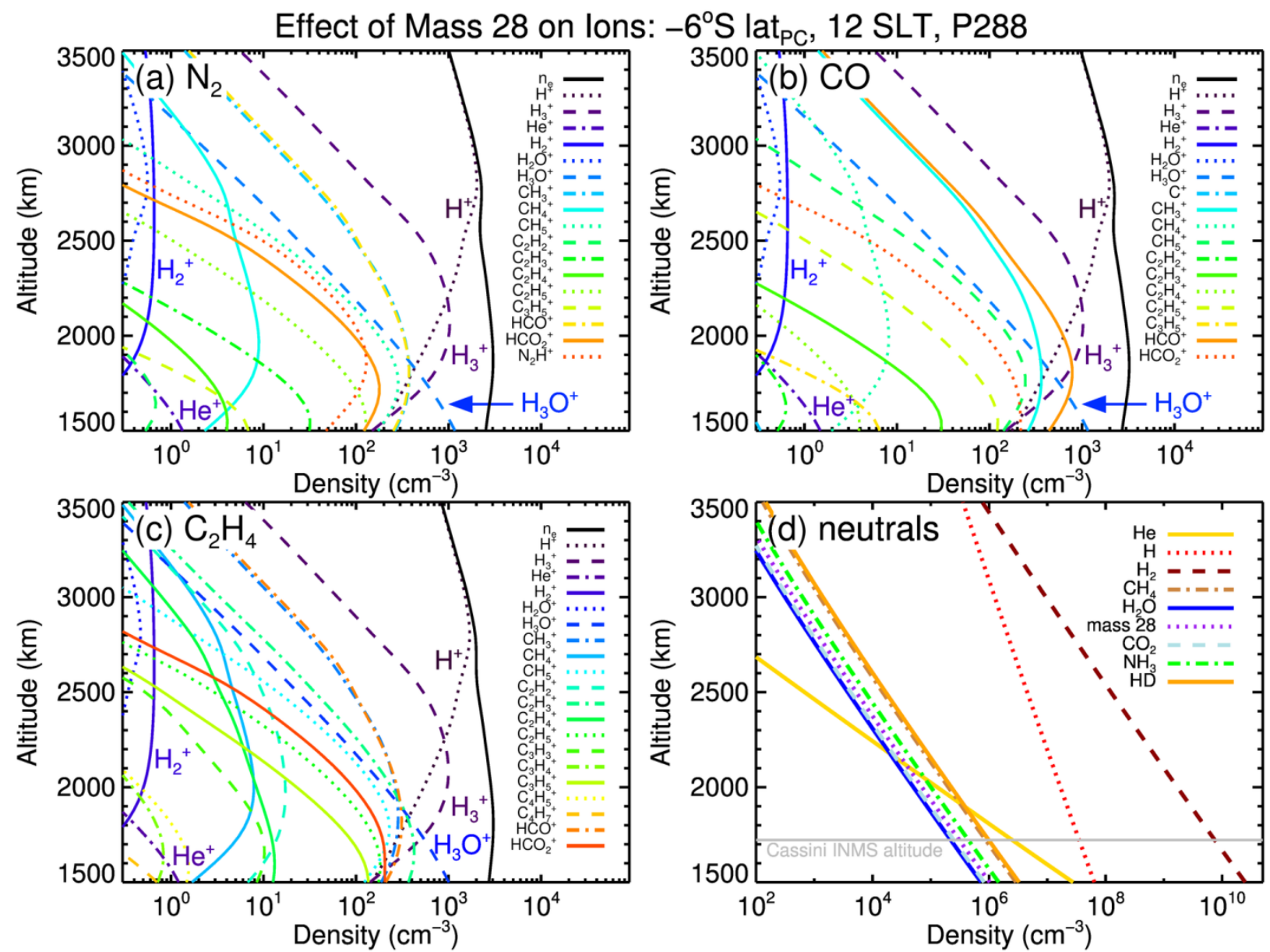

Figure 2: Model results at $-6^{\circ}$ latitude, local solar noon. The density of the 28 Da neutral species is fixed at $40 \%$ of the measured methane density, and it is assumed to be entirely (a) $\mathrm{N}_{2}$, (b) $\mathrm{CO}$, or (c) $\mathrm{C}_{2} \mathrm{H}_{4}$. Panel (d) presents background neutral constituents used in the modeling.

Modeled $n_{e}$ in each case is comparable owing to the similar dissociative recombination rates of the terminal ions. Similarly, charge-exchange rates between $\mathrm{H}_{3}{ }^{+}$and the candidate $28 \mathrm{Da}$ species are identical to within a factor of $1.6, \mathrm{so}_{3}{ }^{+}$densities are relatively insensitive to the $28 \mathrm{Da}$ neutral(s) and are instead more sensitive to the faster reaction with $\mathrm{H}_{2} \mathrm{O}$. As $\mathrm{H}^{+}$doesn't react with $\mathrm{CO}$ or $\mathrm{N}_{2}$, its density near closest approach appears to be controlled primarily by the 
abundance of $\mathrm{CH}_{4}$ and $\mathrm{H}_{2} \mathrm{O}$, with an additional reduction accompanying any $\mathrm{C}_{2} \mathrm{H}_{4}$. In the presence of so many heavy molecules, the reaction of $\mathrm{H}^{+}$with vibrationally excited $\mathrm{H}_{2}$ is very likely of minor importance: its effective rate here is set to $15 \%$ of the Moses and Bass (2000) value, following Moore et al. (2015). Indeed, $\mathrm{H}^{+}$in Saturn's low-altitude equatorial ionosphere is so effectively controlled by the influx of ring-derived material that the historical situation is close to being reversed: there are now so many paths to $\mathrm{H}^{+}$removal that it is more of a concern to ensure that $\mathrm{H}^{+}$is maintained in the model at the observed $\sim 100-1000 \mathrm{~cm}^{-3}$ level.

\subsection{Model comparisons along Cassini's trajectory}

Model results, extracted from the altitudes, latitudes and local times sampled by Cassini during P288 and P292, are compared with Cassini INMS and RPWS data in Figure 3. Based on analysis of ionization dissociation patterns in INMS high-altitude data, Perry et al. (2018) estimate that $\mathrm{C}_{2} \mathrm{H}_{4}$ comprises $25-30 \%$ of the mass 28 measurement. Therefore, for these simulations we fix $\mathrm{C}_{2} \mathrm{H}_{4}$ at $28 \%$ of the total $28 \mathrm{Da}$ density, and divide the remainder evenly across $\mathrm{N}_{2}$ and $\mathrm{CO}$ (i.e., 36\% each). All other neutrals are specified as in 4.1. While there is uncertainty regarding the exact makeup of the $28 \mathrm{Da}$ species, and indeed, regarding the exact neutral and dust composition in Saturn's ionosphere, these values are well-suited for demonstrating the effect of the exogenic material on the light ions. Given the similar chemical lifetimes for the terminal heavy ions presented in Figure 2, further refinements to the constraints 
on $\mathrm{N}_{2}$ and $\mathrm{CO}$ won't significantly affect the relative heavy-to-light ion mix shown in Figure $\mathbf{3}$ (though they will affect the relative proportions of individual heavy ions).
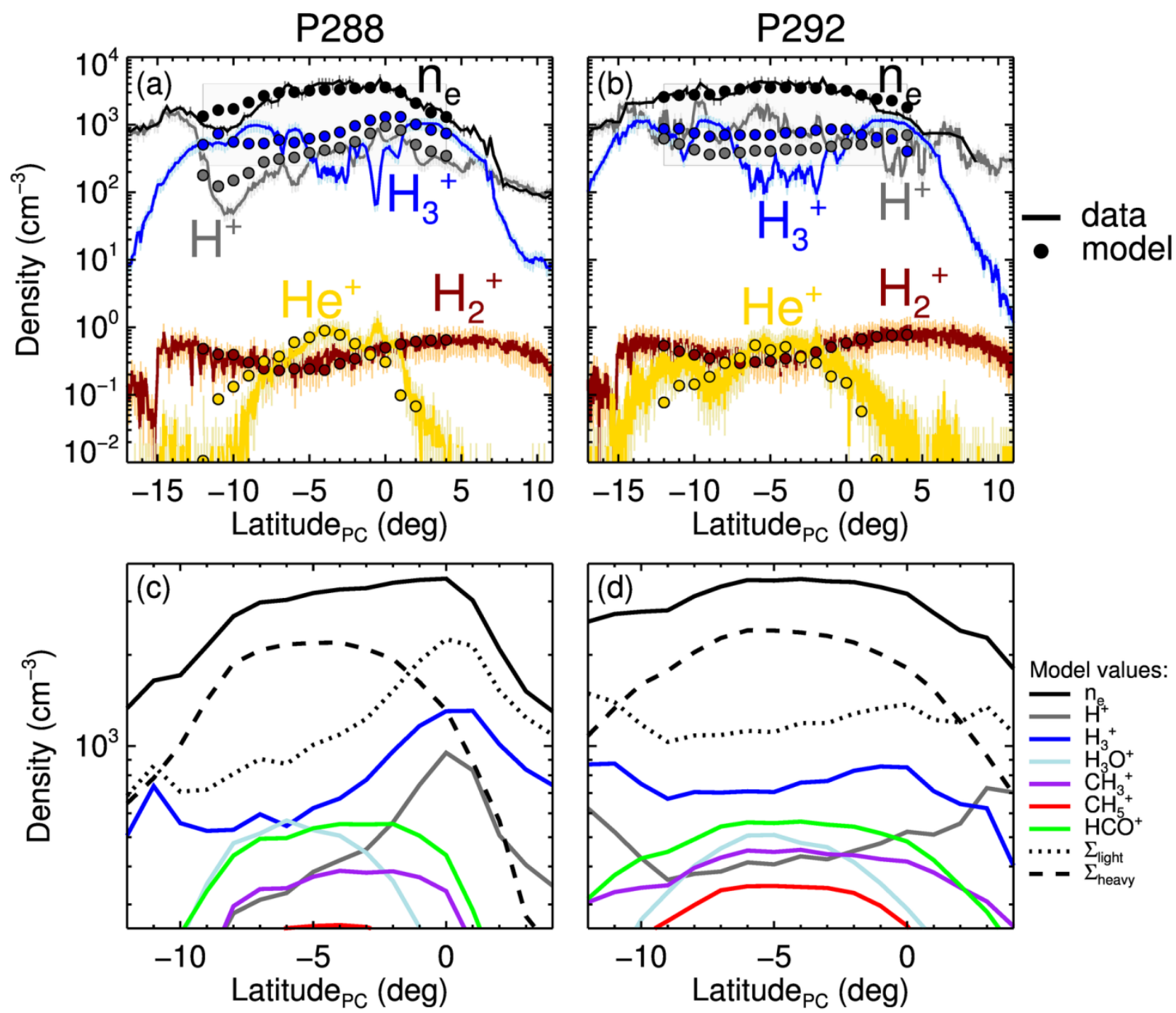

Figure 3: Model (filled circles) comparisons with Cassini observations (solid lines) during (a) P288 and (b) P292, extracted along the spacecraft trajectory. Ion densities come from INMS (Cravens et al., 2018; Waite et al., 2018); electron densities are RPWS results derived from the propagation characteristics of two plasma wave modes (Persoon et al., 2018). Panels (c) and (d) present modeled plasma densities near closest approach for P288 and P292, respectively, as indicated by the gray boxes drawn in (a) and (b). See text for description of model parameters. 
As described in 3.2, the solar flux specified in the model, extrapolated from Earth-based satellite measurements, was enhanced in order to reproduce the observed $\mathrm{H}_{2}^{+}$density at highaltitude. Following that correction, the measured trend in $\mathrm{H}_{2}{ }^{+}$, with a dip near closest approach due to attenuation of solar photons by Saturn's upper atmosphere, is well captured in the model owing to the relatively simple chemistry associated with that ion. Further, neutral densities from the lower end of the range outlined in Figure 1d are used for P292 calculations, in order to better match modeled electron and $\mathrm{He}^{+}$densities. This adjustment corresponded to a roughly $20 \%$ reduction in the non- $\mathrm{H}_{2}$, non-He background neutral densities, well within the given uncertainties. Such $\mathrm{He}^{+}$comparisons may represent an additional means of constraining external influxes, as its density is primarily controlled by reactions with $\mathrm{CH}_{4}$ and candidate mass 28 constituents.

In terms of magnitudes, modeled $\mathrm{H}^{+}, \mathrm{H}_{3}{ }^{+}, \mathrm{He}^{+}$, and $n_{e}$ densities are generally close to observed values (typically within a factor of 2), however there are also a number of structures not reproduced by the model. In particular, there is a prominent structure near $1^{\circ} \mathrm{S}$ that appears as a local maximum in $n_{e}, \mathrm{H}^{+}$and $\mathrm{He}^{+}$, and a local minimum in $\mathrm{H}_{3}{ }^{+}$. This could be the fly-by signature of a narrow electron density layer, frequently seen in radio occultation observations (Nagy et al., 2006), and perhaps caused by gravity waves (Barrow and Matcheva, 2013). No physics that could produce such layers is included in these preliminary 1-D model calculations. The $\mathrm{H}_{3}{ }^{+}$model-data discrepancy between $\sim 5^{\circ} \mathrm{S}$ and $1^{\circ} \mathrm{N}$ is more puzzling. Clearly, modeled $\mathrm{H}_{3}{ }^{+}$ densities are too large. However, the most effective method of removing $\mathrm{H}_{3}{ }^{+}$in this region of 
the ionosphere is by charge-exchange with molecules such as $\mathrm{H}_{2} \mathrm{O}$ and $\mathrm{CH}_{4}$, and these densities are constrained by INMS neutral measurements. Moreover, even if they were increased above the INMS values, they would also further reduce the modeled $\mathrm{H}^{+}$densities, worsening the modeldata agreement for that ion. This requirement - that modeled $\mathrm{H}_{3}{ }^{+}$densities be reduced without affecting $\mathrm{H}^{+}$densities - can be achieved if there is an abundant species present that reacts with $\mathrm{H}_{3}^{+}$, but not $\mathrm{H}^{+}$or $\mathrm{He}^{+}$. The required mixing ratio for such a species is several times larger than the $\sim 10^{-4} \mathrm{CH}_{4}$ mixing ratio and peaks near the equatorial plane (Cravens et al., 2018). None of the measured constituents are known to fit this requirement. It is possible additional chemical pathways may be missing from the model calculations, or that there are additional grain and/or dust-related impacts on $\mathrm{H}_{3}{ }^{+}$densities (e.g., Mitchell et al., 2018; Morooka et al., 2018; Wahlund et al., 2018).

Based on model comparisons to Cassini in situ plasma data, heavy ions (>8 Da) collectively dominate over light ions. Mean ion mass at closest approach for $3 \mathrm{a}$ and $3 \mathrm{~b}$ is calculated to be $11 \mathrm{Da}$, within the range derived from RPWS data (Wahlund et al., 2017; Morooka et al., 2018).

\section{Summary}

The first in situ observations of Saturn's upper atmosphere revealed a transformative contribution from an unexpectedly large and complex composition of in-falling material 
originating in its rings. The impact of that material - which appears to be dominated by organics rather than water as anticipated - on Saturn's ionosphere will take more time to fully understand, but model comparisons with plasma measurements demonstrate that:

- $\quad$ The lower than expected light ion $\left(\mathrm{H}^{+}, \mathrm{H}_{2}{ }^{+}, \mathrm{H}_{3}{ }^{+}, \mathrm{He}^{+}\right)$densities are still broadly consistent with the makeup of the neutral species measured by INMS.

- Molecular ions dominate Saturn's low altitude equatorial ionosphere, with a mean ion mass at closest approach of $11 \mathrm{Da} . \mathrm{H}_{3} \mathrm{O}^{+}$plays an important role, even for reduced levels of water influx. Other dominant heavy ions depend on the unknown makeup of the mass 28 neutral species, but potentially include $\mathrm{HCO}^{+}, \mathrm{N}_{2} \mathrm{H}^{+}, \mathrm{CH}_{3}{ }^{+}, \mathrm{CH}_{5}{ }^{+}$, and $\mathrm{C}_{2} \mathrm{H}_{3}{ }^{+}$.

Remaining mysteries include the explanations behind the 50-70\% solar EUV enhancement required to reproduce observed $\mathrm{H}_{2}^{+}$densities, the observed variability in $\mathrm{H}^{+}$and $\mathrm{H}_{3}^{+}$, and the modeled overabundance in low-altitude $\mathrm{H}_{3}{ }^{+}$. The two latter points require additional processes that reduce $\mathrm{H}_{3}{ }^{+}$without simultaneous $\mathrm{H}^{+}$reduction.

\section{Acknowledgements}

This material is based upon work supported by the National Aeronautics and Space Administration under Grant No. NNX16AI34G issued through the Cassini Data Analysis Program. 


\section{References}

Anicich, V. G. (1993), A survey of bimolecular ion-molecule reactions for use in modeling the chemistry of planetary atmospheres, cometary comae, and interstellar clouds: 1993 supplement, Astrophys. J. Suppl. Ser., (84), 215-315.

Atreya, S. K., T. M. Donahue, A. F. Nagy, J. H. Waite Jr., and J. C. McConnell (1984), Theory, measurements, and models of the upper atmosphere and ionosphere of Saturn, in Saturn, pp. 239-277, University of Arizona Press, Tucson, AZ.

Barrow, D. J., and K. I. Matcheva (2013), Modeling the effect of atmospheric gravity waves on Saturn's ionosphere, Icarus, 224(1), 32-42, doi:10.1016/j.icarus.2013.01.027.

Chen, R. H. (1983), Saturn's ionosphere: A corona of ice particles?, Moon Planets, 28, 37-41.

Connerney, J. (2013), Saturn's ring rain, Nature, 496(7444), 178-179.

Connerney, J., and J. Waite (1984), New model of Saturn's ionosphere with an influx of water from the rings, Nature, 312, 136-138.

Cravens, T. E. (1987), Vibrationally Excited Molecular Hydrogen in the Upper Atmosphere of Jupiter, J. Geophys. Res., 92(5), 11,083-11,100.

Cravens, T. E., L. Moore, J. H. Waite Jr., R. Perryman, M. Perry, J.-E. Wahlund, A. Persoon, and W. Kurth (2018), The ion composition of Saturn's equatorial ionosphere as observed by Cassini, Geophys. Res. Lett., submitted, $1-16$.

Cuzzi, J. N. et al. (2010), An evolving view of Saturn's dynamic rings., Science, 327(5972), 1470-5, doi:10.1126/science.1179118.

Feuchtgruber, H., E. Lellouch, and T. De Graauw (1997), External supply of oxygen to the atmospheres of the giant planets, Nature, 389(September), 159-162.

Galand, M., L. Moore, B. Charnay, I. Mueller-Wodarg, and M. Mendillo (2009), Solar primary and secondary ionization at Saturn, J. Geophys. Res., 114(A6), A06313, doi:10.1029/2008JA013981.

Girazian, Z., and P. Withers (2015), An empirical model of the extreme ultraviolet solar spectrum as a function of F10.7, J. Geophys. Res. Sp. Phys., 120, 6779-6794, doi:10.1002/2015JA021436.

Hadid, L. Z. et al. (2018), Ring shadowing effects on Saturn's ionosphere: Implications for ring opacity and plasma transport, Geophys. Res. Lett., submitted, 1-16.

Hsu, H. et al. (2018), Cosmic Dust Analyzer onboard Cassini Collects Material from Saturn's Main Rings, Science (80-. )., submitted, 1-28.

Huang, J., Y. Hao, D. Zhang, and Z. Xiao (2017), Revisiting interminima solar EUV change using adjusted SOHO SEM data, J. Geophys. Res. A Sp. Phys., 122, 3420-3429, doi:10.1002/2016JA023664.Abstract.

Kim, Y. H., J. L. Fox, J. H. Black, and J. I. Moses (2014), Hydrocarbon ions in the lower ionosphere of Saturn, J. Geophys. Res. Sp. Phys., 119, 1-12, doi:10.1002/2013JA019022.

Kliore, A. J., I. R. Patel, G. F. Lindal, D. N. Sweetnam, and H. B. Hotz (1980), Structure of the Ionosphere and Atmosphere of Saturn From Pioneer 11 Saturn Radio Occultation, J. Geophys. Res., 85(A11), 5857-5870.

Kliore, A. J., A. F. Nagy, E. A. Marouf, A. Anabtawi, E. Barbinis, D. U. Fleischman, and D. S. Kahan (2009), Midlatitude and high-latitude electron density profiles in the ionosphere of Saturn obtained by Cassini radio occultation observations, J. Geophys. Res., 114(A4), A04315, doi:10.1029/2008JA013900.

Kliore, A. J., A. Nagy, S. Asmar, A. Anabtawi, E. Barbinis, D. Fleischman, D. Kahan, J. Klose, and K. E. T. Al (2014), The ionosphere of Saturn as observed by the Cassini Radio Science System, Geophys. Res. Lett., 4l(16), 1-5, doi:10.1002/2014GL060512.Received. 
Lindal, G. F., D. N. Sweetnam, and V. R. Eshleman (1985), The Atmosphere of Saturn: An Analysis of the Voyager Radio Occultation Measurements, Astron. J., 90(6), 1136-1146.

Majeed, T., and J. McConnell (1991), The upper ionospheres of Jupiter and Saturn, Planet. Space Sci., 39(12), $1715-1732$.

Majeed, T., J. McConnell, and R. Yelle (1991), Vibrationally excited H2 in the outer planets thermosphere: Fluorescence in the Lyman and Werner bands, Planet. Space Sci., 39(11), 1591-1606.

McElroy, M. (1973), The ionospheres of the major planets, Space Sci. Rev., 14, 460-473.

Mitchell, D. G. et al. (2018), D-Ring Dust Falling into Saturn's Equatorial Upper Atmosphere, Science (80-. )., submitted, 1-18.

Moore, L., M. Galand, I. Mueller-Wodarg, and M. Mendillo (2009), Response of Saturn's ionosphere to solar radiation: Testing parameterizations for thermal electron heating and secondary ionization processes, Planet. Space Sci., 57(14-15), 1699-1705, doi:10.1016/j.pss.2009.05.001.

Moore, L., I. Mueller-Wodarg, M. Galand, A. Kliore, and M. Mendillo (2010), Latitudinal variations in Saturn's ionosphere: Cassini measurements and model comparisons, J. Geophys. Res., 115(A11), A11317, doi:10.1029/2010JA015692.

Moore, L., J. O’Donoghue, I. Müller-Wodarg, M. Galand, and M. Mendillo (2015), Saturn ring rain: Model estimates of water influx into Saturn's atmosphere, Icarus, 245, 355-366, doi:10.1016/j.icarus.2014.08.041.

Moore, L., M. Galand, A. J. Kliore, A. F. Nagy, and J. O 'Donoghue (2017), Saturn's Ionosphere, in Saturn in the 21 st Century, edited by K. H. Baines, F. M. Flasar, N. Krupp, and T. S. Stallard, p. arXiv:1701.05178, Cambridge University Press, Cambridge.

Moore, L. E., M. Mendillo, I. C. F. Müller-Wodarg, and D. L. Murr (2004), Modeling of global variations and ring shadowing in Saturn's ionosphere, Icarus, 172(2), 503-520, doi:10.1016/j.icarus.2004.07.007.

Morooka, M. W. et al. (2018), Saturn's Dusty Ionosphere of Heavy Ions, Geophys. Res. Lett., submitted, 1-13.

Moses, J., and S. Bass (2000), The effects of external material on the chemistry and structure of Saturn's ionosphere, J. Geophys. Res., 105(1999), 7013-7052.

Moses, J. I., and A. R. Poppe (2017), Dust Ablation on the Giant Planets: Consequences for Stratospheric Photochemistry, Icarus, (297), 33-58, doi:10.1016/j.icarus.2017.06.002.

Müller-Wodarg, I. C. F., L. Moore, M. Galand, S. Miller, and M. Mendillo (2012), Magnetosphere-atmosphere coupling at Saturn: 1 - Response of thermosphere and ionosphere to steady state polar forcing, Icarus, 221(2), 481-494, doi:10.1016/j.icarus.2012.08.034.

Nagy, A. F. et al. (2006), First results from the ionospheric radio occultations of Saturn by the Cassini spacecraft, $J$. Geophys. Res., 111(A6), A06310, doi:10.1029/2005JA011519.

O’Donoghue, J., T. S. Stallard, H. Melin, G. H. Jones, S. W. H. Cowley, S. Miller, K. H. Baines, and J. S. D. Blake (2013), The domination of Saturn's low-latitude ionosphere by ring "rain," Nature, 496(7444), 193-195, doi:10.1038/nature12049.

O’Donoghue, J., L. Moore, J. E. P. Connerney, H. Melin, T. S. Stallard, S. Miller, and K. H. Baines (2017), Redetection of the ionospheric H3+ signature of Saturn's "ring rain," Geophys. Res. Lett., 1-8, doi:10.1002/2017GL075932.

Perry, M. E. et al. (2018), Material Flux from the Rings of Saturn into its Atmosphere, Geophys. Res. Lett., submitted.

Persoon, A. M. et al. (2018), Electron density distributions in Saturn's ionosphere, Geophys. Res. Lett., submitted, $1-17$.

Poppe, A. R. (2016), An improved model for interplanetary dust fluxes in the outer Solar System, Icarus, 264, 369386, doi:10.1016/j.icarus.2015.10.001. 
Prangé, R., T. Fouchet, R. Courtin, J. E. P. Connerney, and J. C. McConnell (2006), Latitudinal variation of Saturn photochemistry deduced from spatially-resolved ultraviolet spectra, Icarus, 180(2), 379-392, doi:10.1016/j.icarus.2005.11.005.

Schunk, R. W., and A. F. Nagy (2009), Ionospheres: Physics, Plasma Physics, and Chemistry, 2nd ed., Cambridge University Press, Cambridge, UK.

Shimizu, M. (1980), Strong interaction between the ring system and the ionosphere of Saturn, Moon Planets, 22, $521-522$.

Wahlund, J. et al. (2017), In situ measurements of Saturn's ionosphere show that it is dynamic and interacts with the rings, Science (80-. )., 4134(December), 1-8, doi:10.1029/1999JE001172.

Wahlund, J. -E. et al. (2018), On the Characteristics of Charged Dust in Saturn's Equatorial Ionosphere Implications from Cassini RPWS/LP data, Geophys. Res. Lett., submitted, 1-12.

Waite, J. H., S. K. Atreya, and A. F. Nagy (1979), The ionosphere of Saturn: predictions for Pioneer 11, Geophys. Res. Lett., 6(9), 723-726.

Waite, J. H. et al. (2006), Cassini ion and neutral mass spectrometer: Enceladus plume composition and structure, Science (80-. )., 311(5766), 1419-22, doi:10.1126/science.1121290.

Waite, J. H. J. et al. (2018), INMS observations of chemical interactions between Saturn's atmosphere and rings, Science (80-. )., submitted, 1-38.

Winkelstein, P., J. Caldwell, S. J. Kim, G. E. Hunt, and V. Moore (1983), A Determination of the Composition of the Saturnian Stratosphere Using the IUE, Icarus, 54, 309-318.

Woods, T. N., F. G. Eparvier, S. M. Bailey, P. C. Chamberlin, J. Lean, G. J. Rottman, S. C. Solomon, W. K. Tobiska, and D. L. Woodraska (2005), Solar EUV Experiment (SEE): Mission overview and first results, $J$. Geophys. Res., 110(A1), A01312, doi:10.1029/2004JA010765.

Yelle, R. V, J. Serigano, T. T. Koskinen, S. M. Horst, M. E. Perry, R. S. Perryman, and J. H. J. Waite (2018), Thermal Structure and Composition of Saturn's Upper Atmosphere from Cassini/INMS Measurements, Geophys. Res. Lett., submitted, 1-18. 